\title{
Simplification of Coefficients in Two Families of Nonlinear Ordinary Differential Equations
}

\author{
Feng Qi ${ }^{1,2,3, *}$, Bai-Ni Guo ${ }^{4}$ \\ ${ }^{1}$ Institute of Mathematics, Henan Polytechnic University, Jiaozuo, Henan, 454010, China
${ }^{2}$ College of Mathematics, Inner Mongolia University for Nationalities, Tongliao, Inner Mongolia, 028043, China \\ ${ }^{2}$ Department of Mathematics, College of Science, Tianjin Polytechnic University, Tianjin, 300387, China \\ ${ }^{4}$ School of Mathematics and Informatics, Henan Polytechnic University, Jiaozuo, Henan, 454010, China \\ *Corresponding author: qifeng618@gmail.com,
}

Received April 29, 2018; Revised June 02, 2018; Accepted July 27, 2018

\begin{abstract}
In the paper, in terms of the Stirling numbers of the first and second kinds, by three approaches, the author derives simple, meaningful, and significant forms for coefficients in two families of nonlinear ordinary differential equations.
\end{abstract}

Keywords: coefficient, nonlinear ordinary differential equation, Stirling number, derivative polynomial

Cite This Article: Feng Qi, and Bai-Ni Guo, "Simplification of Coefficients in Two Families of Nonlinear Ordinary Differential Equations." Turkish Journal of Analysis and Number Theory, vol. 6, no. 4 (2018): 116-119. doi: 10.12691/tjant-6-4-2.

\section{Motivation and Main Results}

In [[6], Theorems 2.1 and 2.2], it was established inductively and recursively that the function

$$
F(t)=\frac{1}{2-e^{t}}
$$

satisfies the families of nonlinear ordinary differential functions

$$
F^{(n)}(t)=\sum_{k=0}^{n}(-1)^{n-k} a_{k}(n) F^{k+1}(t)
$$

and

$$
2^{n} n ! F^{n+1}(t)=\sum_{k=0}^{n} b_{k}(n) F^{(k)}(t)
$$

for $n \in \mathbb{N}$, where $a_{0}(n)=1, a_{n}(n)=2^{n} n !, b_{0}(n)=n !$, $b_{n}(n)=1$,

$$
\begin{aligned}
a_{k}(n)= & 2^{k-1} k ! \sum_{i_{k}=0}^{n-k} \sum_{i_{k-1}=0}^{n-k-i_{k}} \ldots \\
& \ldots \sum_{i_{1}=1}^{n-k+1-i_{k}-\cdots-i_{2}} 2^{i_{1}} \cdots k^{i_{k-1}}(k+1)^{i_{k},},
\end{aligned}
$$

and

$$
b_{k}(n)=\sum_{i_{k}=0}^{n-k} \sum_{i_{k-1}=0}^{i_{k}} \ldots \sum_{i_{1}=0}^{i_{2}} \frac{n !}{\prod_{j=1}^{k}\left(i_{j}+j\right)}
$$

for $0<k<n$.
In this paper, since

(1) the original proofs of [[6], Theorems 2.1 and 2.2] are long and tedious,

(2) the expressions in (4) and (5) are too complex to be remembered, understood, and computed easily, we will provide three simple and standard proofs for [[6], Theorems 2.1 and 2.2] and, more importantly, derive simple, meaningful, and significant expressions for the quantities $a_{k}(n)$ and $b_{k}(n)$.

Our main results can be stated as the following theorem. Theorem 1. For $k \in\{0\} \cup \mathbb{N}$, the function $F(t)$ defined by (1) satisfies

$$
F^{(k)}(t)=(-1)^{k} \sum_{m=0}^{k}(-1)^{m} 2^{m} m ! S(k+1, m+1) F^{m+1}(t)(6)
$$

and

$$
F^{k+1}(t)=\frac{(-1)^{k}}{2^{k} k !} \sum_{m=0}^{k}(-1)^{m} s(k+1, m+1) F^{(m)}(t),
$$

where $s(n, k)$ and $S(n, k)$ stand for the Stirling numbers of the first and second kinds.

\section{Proofs of Theorem 1}

In this section, we provide three proofs for Theorem 1 as follows.

First proof. It is well known [[1], Theorem 11.4] and $[[2]$, p. 139, Theorem C] that the Faàdi Bruno formula can be described in terms of the Bell polynomials of the second kind $\mathrm{B}_{n, k}\left(x_{1}, x_{2}, \cdots, x_{n-k+1}\right)$ by 


$$
\begin{aligned}
\frac{\mathrm{d}^{n}}{\mathrm{~d} x^{n}} f^{\circ} h(x)= & \sum_{k=0}^{n} f^{(k)}(h(x)) \\
& \times \mathrm{B}_{n, k}\left(h^{\prime}(x), h^{\prime \prime}(x), \ldots, h^{(n-k+1)}(x)\right) .
\end{aligned}
$$

The identities

$$
\begin{aligned}
& \mathrm{B}_{n, k}\left(a b x_{1}, a b^{2} x_{2}, \ldots, a b^{n-k+1} x_{n-k+1}\right) \\
& =a^{k} b^{n} \mathrm{~B}_{n, k}\left(x_{1}, x_{2}, \ldots, x_{n-k+1}\right)
\end{aligned}
$$

and

$$
\mathrm{B}_{n, k}(1,1, \ldots, 1)=S(n, k)
$$

for $n \geq k \geq 0$ and $a, b \in \mathbb{C}$ can be found in [[1],p. 412] and [[2], p. 135]. Applying (8), (9), and (10) in sequence and denoting $u=u(x)=2-e^{x}$ yield

$$
\begin{aligned}
& F^{(n)}(t)=\sum_{k=0}^{n}\left(\frac{1}{u}\right)^{(k)} \mathrm{B}_{n, k}\left(-e^{x},-e^{x}, \ldots,-e^{x}\right) \\
&=\sum_{k=0}^{n} \frac{(-1)^{k} k !}{u^{k+1}}\left(-e^{x}\right)^{k} \mathrm{~B}_{n, k}(1,1, \ldots, 1) \\
&=\sum_{k=0}^{n} \frac{(-1)^{k} k !}{\left(2-e^{x}\right)^{k+1}}\left[\left(2-e^{x}\right)-2\right]^{k} S(n, k) \\
&=\sum_{k=0}^{n} \frac{(-1)^{k} k !}{\left(2-e^{x}\right)^{k+1}} S(n, k) \sum_{\ell=0}^{k}\left(\begin{array}{l}
k \\
\ell
\end{array}\right)\left(2-e^{x}\right)^{\ell}(-2)^{k-\ell} \\
&=\sum_{k=0}^{n} k ! S(n, k) \sum_{\ell=0}^{k}(-1)^{\ell}\left(\begin{array}{c}
k \\
\ell
\end{array}\right) F^{k-\ell+1}(x) 2^{k-\ell} \\
&=\sum_{k=0}^{n}(-1)^{k} k ! S(n, k) \sum_{\ell=0}^{k}(-1)^{\ell}\left(\begin{array}{c}
k \\
\ell
\end{array}\right) F^{\ell+1}(x) 2^{\ell} \\
&= \sum_{\ell=0}^{n}(-1)^{\ell} F^{\ell+1}(x) 2^{\ell} \sum_{k=\ell}^{n}(-1)^{k}\left(\begin{array}{l}
k \\
\ell
\end{array}\right) k ! S(n, k) .
\end{aligned}
$$

Combining this with the identity

$$
\sum_{k=\ell}^{n}(-1)^{k}\left(\begin{array}{l}
k \\
\ell
\end{array}\right) k ! S(n, k)=(-1)^{n} \ell ! S(n+1, \ell+1)
$$

in [[24], Theorem 2.2] results in (6).

The identity (7) follows from applying [[2], p. 213, eq. (5c)] or [[34], p. 171, Theorem 12.1], which reads that

$$
a_{n}=\sum_{\alpha=0}^{n} S(n, \alpha) b_{\alpha} \text { if and only if } b_{n}=\sum_{k=0}^{n} s(n, k) a_{k}
$$

for a collection of constants $b_{\alpha}$ and $a_{k}$ independent of $n$, to (6). The first proof of Theorem 1 is complete.

Second proof. A sequence of polynomials $P_{n}$ of order $n$ are called $[5,9,22]$ the derivative polynomials of a function $f(x)$ if and only if $f^{(n)}(x)=P_{n}(f(x))$ for $n \geq 0$. In [[38], Theorem 1.1], it was obtained that the derivative polynomials of the function $F(t, \lambda)=\frac{1}{e^{t}+\lambda}$ can be computed by

$$
P_{n}(x)=\sum_{k=0}^{n}(-1)^{n-k} k ! S(n+1, k+1) \lambda^{k} x^{k+1}
$$

for $\lambda \neq 0$ and $n \in \mathbb{N}$. In [[38], Theorem 1.3], it was obtained that the nonlinear differential equations

$$
\begin{aligned}
& \sum_{k=0}^{n}(-1)^{k} s(n+1, k+1) \frac{\mathrm{d}^{k} F(t, \lambda)}{\mathrm{d} t^{k}} \\
& =(-1)^{n} n ! \lambda^{n} F^{n+1}(t, \lambda)
\end{aligned}
$$

have a common solution $F(t, \lambda)=\frac{1}{e^{t}+\lambda}$ for $\lambda \neq 0$ and $n \in \mathbb{N}$. Letting $\lambda=-2$ in (12) and (13) gives

$$
P_{n}(x)=(-1)^{n} \sum_{k=0}^{n} k ! S(n+1, k+1) 2^{k} x^{k+1}
$$

and

$$
\sum_{k=0}^{n}(-1)^{k} s(n+1, k+1) F^{(k)}(t)=n ! 2^{n} F^{n+1}(t) .
$$

The second proof of Theorem 1 is thus complete.

Third proof. By virtue of Theorem 2.1 in [3], Theorems 3.1 and 3.2 in [37], and Lemma 2.1 in [38], it follows that

$$
\begin{aligned}
& \frac{\mathrm{d}^{k}}{\mathrm{~d} t^{k}}\left(\frac{1}{\beta e^{\alpha t}-1}\right) \\
& =(-1)^{k} \alpha^{k} \sum_{m=1}^{k+1}(m-1) ! S(k+1, m)\left(\frac{1}{\beta e^{\alpha t}-1}\right)^{m}
\end{aligned}
$$

and

$$
\begin{aligned}
\left(\frac{1}{\beta e^{\alpha t}-1}\right)^{k}= & \frac{1}{(k-1) !} \sum_{m=1}^{k} \frac{(-1)^{m-1}}{\alpha^{m-1}} \\
& \times s(k, m) \frac{\mathrm{d}^{m-1}}{\mathrm{~d} t^{m-1}}\left(\frac{1}{\beta e^{\alpha t}-1}\right),
\end{aligned}
$$

where $\alpha, \beta \neq 0$ are real constants, $k \in \mathbb{N}$, either $\beta>0$ and $t \neq-\frac{\ln \beta}{\alpha}$ or $\beta<0$ and $t \in \mathbb{R}$. See also $[4,35,36]$.

Taking $\alpha=1$ and $\beta=\frac{1}{2}$ in (14) and (15) leads to

$$
\begin{aligned}
& \frac{\mathrm{d}^{k}}{\mathrm{~d} t^{k}}\left(\frac{1}{e^{t} / 2-1}\right) \\
& =(-1)^{k} \sum_{m=1}^{k+1}(m-1) ! S(k+1, m)\left(\frac{1}{e^{t} / 2-1}\right)^{m}
\end{aligned}
$$

and

$$
\begin{aligned}
& \left(\frac{1}{e^{t} / 2-1}\right)^{k} \\
& =\frac{1}{(k-1) !} \sum_{m=1}^{k}(-1)^{m-1} s(k, m) \frac{\mathrm{d}^{m-1}}{\mathrm{~d} t^{m-1}}\left(\frac{1}{e^{t} / 2-1}\right) .
\end{aligned}
$$

These two identities can be further rearranged as 


$$
\begin{aligned}
-2 \frac{\mathrm{d}^{k}}{\mathrm{~d} t^{k}}\left(\frac{1}{2-e^{t}}\right)= & (-1)^{k} \sum_{m=1}^{k+1}(-1)^{m} 2^{m}(m-1) ! \\
& \times S(k+1, m)\left(\frac{1}{2-e^{t}}\right)^{m}
\end{aligned}
$$

and

$$
\begin{aligned}
& (-1)^{k} 2^{k}\left(\frac{1}{2-e^{t}}\right)^{k} \\
& =\frac{2}{(k-1) !} \sum_{m=1}^{k}(-1)^{m} s(k, m) \frac{\mathrm{d}^{m-1}}{\mathrm{~d} t^{m-1}}\left(\frac{1}{2-e^{t}}\right) .
\end{aligned}
$$

The third proof of Theorem 1 is thus complete.

\section{Remarks}

Finally, we list several remarks on our main results and closely related things.

Remark 1. Comparing (2) and (3) with (6) and (7) figures out that

$$
a_{m}(k)=2^{m} m ! S(k+1, m+1), \quad k \geq m \geq 0
$$

and

$$
b_{m}(k)=(-1)^{k+m} s(k+1, m+1), \quad k \geq m \geq 0
$$

which are simpler, more meaningful, and more significant than the expressions in (4) and (5).

Remark 2. In [[34], p. 118, Eq. (9.18)], it is listed that

$$
\alpha ! S(n, \alpha)=\sum_{j=\alpha}^{n}(-1)^{n-j}\left(\begin{array}{c}
j-1 \\
\alpha-1
\end{array}\right) j ! S(n, j) .
$$

This identity is different from (11) and

$$
\sum_{\ell=k+1}^{n}(-1)^{\ell}\left(\begin{array}{c}
\ell-1 \\
k
\end{array}\right) \ell ! S(n, \ell)=(-1)^{n}(k+1) ! S(n, k+1)(16)
$$

for $n>k \geq 0$ in [[24], Theorem 2.2].

Remark 3. Any one among three proofs is simpler and shorter than the one in the paper [6].

Remark 4. The motivations in the papers $[3,4,7,8,10,11$, $12,14-33,38]$ are same as the one in this paper.

Remark 5. This paper is a slightly modified version of the preprint [13].

\section{References}

[1] C. A. Charalambides, Enumerative Combinatorics, CRC Press Series on Discrete Mathematics and its Applications. Chapman \& Hall/CRC, Boca Raton, FL, 2002.

[2] L. Comtet, Advanced Combinatorics: The Art of Finite and Infinite Expansions, Revised and Enlarged Edition, D. Reidel Publishing Co., 1974.

[3] B.-N. Guo and F. Qi, Explicit formulae for computing Euler polynomials in terms of Stirling numbers of the second kind, J. Comput. Appl. Math. 272 (2014), 251-257.

[4] B.-N. Guo and F. Qi, Some identities and an explicit formula for Bernoulli and Stirling numbers, J. Comput. Appl. Math. 255 (2014), 568-579.

[5] M. E. Hoffman, Derivative polynomials for tangent and secant, Amer. Math. Monthly 102 (1995), no. 1, 23-30.
[6] G.-W. Jang and T. Kim, Some identities of ordered Bell numbers arising from differential equation, Adv. Stud. Comtemp. Math. 27 (2017), no. 3, 385-397.

[7] F. Qi, A simple form for coefficients in a family of nonlinear ordinary differential equations, Adv. Appl. Math. Sci. 17 (2018), no. 8, 555-561.

[8] F. Qi, A simple form for coefficients in a family of ordinary differential equations related to the generating function of the Legendre polynomials, Adv. Appl. Math. Sci. 17 (2018), in press; ResearchGate Preprint (2017).

[9] F. Qi, Derivatives of tangent function and tangent numbers, Appl. Math. Comput. 268 (2015), 844-858.

[10] F. Qi, Explicit formulas for the convolved Fibonacci numbers, ResearchGate Working Paper (2016).

[11] F. Qi, Notes on several families of differential equations related to the generating function for the Bernoulli numbers of the second kind, Turkish J. Anal. Number Theory 6 (2018), no. 2, 40-42.

[12] F. Qi, Simple forms for coefficients in two families of ordinary differential equations, Glob. J. Math. Anal. 6 (2018), no. 1, 7-9.

[13] F. Qi, Simplification of coefficients in two families of nonlinear ordinary differential equations, ResearchGate Preprint (2017).

[14] F. Qi, Simplifying coefficients in a family of nonlinear ordinary differential equations, Acta Comment. Univ. Tartu. Math. (2018), in press; ResearchGate Preprint (2017).

[15] F. Qi, Simplifying coefficients in a family of ordinary differential equations related to the generating function of the Laguerre polynomials, ResearchGate Preprint (2017).

[16] F. Qi, Simplifying coefficients in a family of ordinary differential equations related to the generating function of the Mittag-Leffler polynomials, ResearchGate Preprint (2017).

[17] F. Qi, Simplifying coefficients in differential equations related to generating functions of reverse Bessel and par-tially degenerate Bell polynomials, Bol. Soc. Paran. Mat. (2019), in press; ResearchGate Preprint (2017).

[18] F. Qi and B.-N. Guo, A diagonal recurrence relation for the Stirling numbers of the first kind, Appl. Anal. Discrete Math. 12 (2018), no. 1, 153-165.

[19] F. Qi and B.-N. Guo, Explicit formulas and recurrence relations for higher order Eulerian polynomials, Indag. Math. 28 (2017), no. 4, 884-891.

[20] F. Qi and B.-N. Guo, Explicit formulas for derangement numbers and their generating function, J. Nonlinear Funct. Anal. 2016, Article ID 45, 10 pages.

[21] F. Qi and B.-N. Guo, Some properties of the Hermite polynomials and their squares and generating functions, Preprints 2016, 2016110145, 14 pages.

[22] F. Qi and B.-N. Guo, Viewing some ordinary differential equations from the angle of derivative polynomials, Iran. J. Math. Sci. Inform. 14 (2019), no. 2, in press; Preprints2016, 2016100043 , 12 pages.

[23] F. Qi, D. Lim, and B.-N. Guo, Explicit formulas and identities for the Bell polynomials and a sequence of polynomials applied to differential equations, Rev. R. Acad. Cienc. Exactas Fís. Nat. Ser. A Mat. RACSAM (2018), in press.

[24] F. Qi, D. Lim, and B.-N. Guo, Some identities related to Eulerian polynomials and involving the Stirling numbers, Appl. Anal. Discrete Math. 12 (2018), no. 2, in press.

[25] F. Qi, D.-W. Niu, and B.-N. Guo, Simplification of coefficients in differential equations associated with higher order FrobeniusEuler numbers, Preprints 2017, 2017080017, 7 pages.

[26] F. Qi, D.-W. Niu, and B.-N. Guo, Simplifying coefficients in differential equations associated with higher or-der Bernoulli numbers of the second kind, Preprints 2017, 2017080026, 6 pages.

[27] F. Qi, D.-W. Niu, and B.-N. Guo, Some identities for a sequence of unnamed polynomials connected with the Bell polynomials, Rev. R. Acad. Cienc. Exactas Fís. Nat. Ser. A Math. RACSAM 112 (2018), in press.

[28] F. Qi, X.-L. Qin, and Y.-H. Yao, The generating funtion of the Catalan numbers and lower triangular integer matrices, Preprints 2017, 2017110120, 12 pages.

[29] F. Qi, J.-L. Wang, and B.-N. Guo, Notes on a family of inhomogeneous linear ordinary differential equations, Adv. Appl. Math. Sci. 17 (2018), no. 4, 361-368.

[30] F. Qi, J.-L. Wang, and B.-N. Guo, Simplifying and finding nonlinear ordinary differential equations, ResearchGate Working Paper (2017). 
[31] F. Qi, J.-L. Wang, and B.-N. Guo, Simplifying differential equations concerning degenerate Bernoulli and Euler numbers, Trans. A. Razmadze Math. Inst. 172 (2018), no. 1, 90-94.

[32] F. Qi and J.-L. Zhao, Some properties of the Bernoulli numbers of the second kind and their generating function, Bull. Korean Math. Soc. 55 (2018).

[33] F. Qi, Q. Zou, and B.-N. Guo, Some identities and a matrix inverse related to the Chebyshev polynomials of the second kind and the Catalan numbers, Preprints 2017, 2017030209, 25 pages.

[34] J. Quaintance and H. W. Gould, Combinatorial Identities for Stirling Numbers, the unpublished notes of H. W. Gould, with a foreword by George E. Andrews, World Scientific Publishing Co. Pte. Ltd., Singapore, 2016.
[35] C.-F. Wei and B.-N. Guo, Complete monotonicity of functions connected with the exponential function and derivatives, Abstr. Appl. Anal. 2014 (2014), Article ID 851213, 5 pages.

[36] A.-M. Xu and G.-D. Cen, Closed formulas for computing higherorder derivatives of functions involving exponential functions, Appl. Math. Comput. 270 (2015), 136-141.

[37] A.-M. Xu and Z.-D. Cen, Some identities involving exponential functions and Stirling numbers and applications, J. Comput. Appl. Math. 260 (2014), 201-207.

[38] J.-L. Zhao, J.-L. Wang, and F. Qi, Derivative polynomials of a function related to the Apostol-Euler and Frobenius-Euler numbers, J. Nonlinear Sci. Appl. 10 (2017), no. 4, 1345-1349. 